\title{
Study of Separation Control of Vortex Generators on Transonic Wings*
}

\author{
Jingbo HUANG**, Song FU**, Zhixiang XIAO** and Miao ZHANG*** \\ ** School of Aerospace Engineering, Tsinghua University \\ Haidian District, Beijing, China, 100084 \\ E-mail:hejob0104@163.com \\ *** Shanghai Aircraft Design and Research Institute of COMAC
}

Shanghai, China, 200232

\begin{abstract}
Flows around vortex generators (VGs), which serve as one of the important flow control methods, are investigated by solving Reynolds-Averaged Navier-Stokes (RANS) equations. The influences on the main flow of VGs are intended to explore. To validate computational schemes, the flow around a single VG on a flat plane is computed to acquire basic knowledge of this kind of flow. Then transonic flow past a standard model, named by ONERA-M6 wing, is predicted to investigate the flow features of shockwave/boundary-layer interactions (SWBLI). Investigation is focused on a supercritical wing. Firstly, the effects of a row of VGs on the airfoil with the same cross-section design are calculated with periodical boundaries in transonic conditions. Then, VGs on the whole supercritical wing are analyzed with strong SWBLI. Lastly, VGs are mounted more upwind (about 3.5\% local chord) to explore the effects at low speed and high incidence condition. The numerical results show that seven VGs on the wing can effectively suppress the separations behind the strong SWBLI and decrease spanwise flow and wing-tip vortex in transonic condition. VGs can also decrease the large scope of separation over the wing at low speed with high angle of attack.
\end{abstract}

Key words: Supercritical Wing, Vortex Generator (VG), Shockwave/Boundary-Layer Interactions (SWBLI), Separation Control, Transonic

\section{Introduction}

Vortex generators (VGs) are typical passive flow control devices which can be traced back to mid of the 20th century. The usual shape is an aerodynamic surface which can generate longitudinal vortices to give rise to local mixing of mean flow, to energize the low-energy boundary layer and to delay or prevent the turbulent boundary layer separation. The primary objective using VGs is to decrease drag and increase lift, if possible.

VGs have been applied in the practical airplane for several decades. In fact, on an air-journey by Boeing civil transport series, such as 737-800, 8 pieces of VGs in line near the leading edge are clearly visible. At the same time, 12 and 16 pieces of VGs are mounted on the upper wing of Boeing 757 and 777, respectively. Among Boeing 737, 757 and 777, the VGs look like in line and they are mounted in the midspan of the wing. However, the VGs can be also arranged on the upper surface of wing near the tip, such as Golf-Stream. Fig. 1 presents VGs on the wing of Boeing 737-800 and Golf-Stream, respectively.

The aerodynamic design of wing for the civil transport is the key technologies, which [DOI: 10.1299/fst.6.85]

Copyright $\odot 2011$ by JSME 
increasing lift and reducing drag are the invariable targets for civil transport design, because they are the two key factors which can estimate if the airplane is successful or not. The optimization design of wing configuration can satisfy the needs in most of typical conditions. However, it is very difficult to obtain further improvements based on the optimized wing configuration at some special states, due to many limits, such as structure, weight, and so on. This work focuses on the design of special instruments, like VGs, used to improve wing performance in certain conditions almost without baseline geometry modification.

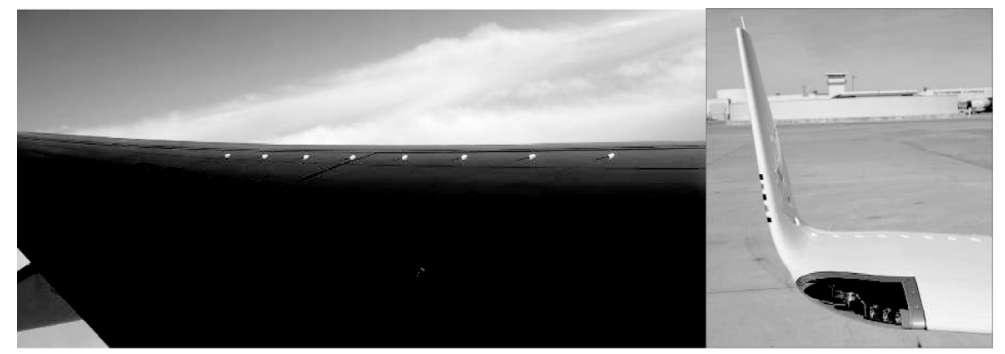

Figure 1 VGs on upper surface of wing on Boeing-737-800 and Golf-Stream.

However, VG's design strongly depends on engineering experiences, because the mechanism between the vortices induced by VGs and boundary layer is not still well known. There are large numbers of combinations of VG parameters, such as surface shape, interval between VGs, arrangement style, deflection angle, and so on. The main difficulty for VGs' design by experiment and simulations is the distinct scale difference between VG and practical wing. (i) The experimental wing model in wind-tunnel is usually $1 / 10$, or even $1 / 20$ times of the original size, while the real sizes of VGs are only several centimeters. After scaling, the size of VG is too small to assure reliability of experimental results. (ii) High precision of measurement is desired as VG's height is on the same order of boundary-layer thickness, especially for the experimental wing model. (iii) In numerical predictions, large amount of grids is needed in normal and spanwise directions of VG to predict flow in more detail. Thus, computational efficiency is hardly ensured. For these reasons, there are few studies about VGs on real wings in references. Vos et al. ${ }^{(1)}$ gave a broad perspective of the progress in solving NS (Navier-Stokes) equations and listed some numerical results about VGs on a BAe Hawk wing using a CFD software FINFLO. Small local disturbances generated by the vortex generators change the overall flow field by moving downward the location of a shock wave. Liu et al. ${ }^{(2)}$ numerically predicted flow around a supercritical wing with VGs using S-A turbulence model and multi-block structured grid (about $5 \mathrm{M}$ grid cells). In their paper, the influences of VGs at low-speed and transonic states were presented. However, they did not deeply investigate the shockwave/boundary-layer interactions (SWBLIs).

As mentioned before, although VGs are applied in the practical airplane, the mechanism is not clear, and reliable design rules are still missing due to great experimental and computational difficulties. Some measurements, computations and statistical models about VGs are available in open literatures. $\mathrm{Ni}^{(3)}$ tested different important parameters of VGs, and discovered that VGs could efficiently reduce boundary layer thickness. Lin $^{(4)}$ reviewed the research of low-profile VG and found that VG would function well in flows with relatively static separation point if VGs were laid in separation zones. Lin et al. ${ }^{(5),(6)}$ tested a single VG in plane channel flows with particle image velocimetry (PIV) and

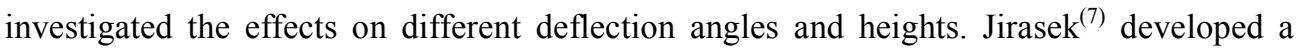
new VG model, named jBAY, which provides an efficient method for CFD simulation of flow systems with vortex generator arrays. $\operatorname{Erik}^{(8)}$ numerically analyzed flow behind VG on a plane with Spalart-Allmaras (SA), $k$ - $\omega$-SST (shear stress transport) and RSM (Reynolds 
stress model) turbulence models, and found that RSM model predicted better than traditional eddy viscosity models based on homogeneous isotropic turbulence hypothesis. Zhang $^{(9)}$ experimentally studied micro-VG's control effect on a supercritical airfoil boundary-layer separation in low-speed wind tunnel. Vavilis and Ekaterinaris ${ }^{(10)}$ predicted the passive and active flow control concepts, which can suppress separated flow and increase the aerodynamic performance of the wing. Florian ${ }^{(11)}$ developed a statistical VG model based on CFD simulation and experiments and achieved acceptable results.

In fact, in supercritical wing designs, flows around VGs mounted on the wing also need to be investigated by numerical simulations. Computational methods, which have both efficiency and precision, are suitable to predict the flows around VGs on the wing and it will help improve the performance of the large civil transport. In this paper, firstly, flows past a single VG on the flat plane and a clear ONERA-M6 wing are discussed as verifications of numerical methods. Then, we focus our study on the effect of VGs in-line mounted on a realistic supercritical wing in transonic condition and low-speed conditions.

\section{Numerical Methods}

The computations here are all based on a compressible solver by solving Reynolds-averaged Navier-Stokes (RANS) equations in a cell-centered finite-volume formulation with multi-block structured grids. The spatial schemes for the convection of NS equations include Jameson's type centered scheme with artificial dissipation, flux-vector-splitting type van Leer and flux-difference-splitting type Roe schemes, and so on. Higher-order schemes are obtained for the upwind schemes through MUSCL or WENO interpolations. The diffusions are solved using central difference.

A modified fully implicit LU-SGS with Newton-like sub-iteration in pseudo time is taken as the time-marching method when solving the mean flow equations and the turbulence model equations.

Turbulence models available in the in-house code ${ }^{(12),(13)}$ are SA, $k-g, k-\omega-\mathrm{SST}$, $k$ - $\omega$-Wilcox-1988/1998/2006 models and so on. Large Eddy Simulation (LES) method with Smagrinsky subgrid stress model and RANS/LES hybrid methods ${ }^{(14)-(17)}$ based on some turbulence models are also available. Turbulence equations are solved using similar time-marching and spatial discretization schemes as mean flow equations, and positive items of production are explicitly treated while negative items of destruction terms are implicitly solved.

In this article, Roe scheme with the 3rd-order MUSCL or the 5th-order WENO and R-S entropy fix ${ }^{(18)}$ is taken as the spatial scheme. LU-SGS without sub-iterations is taken as the time-stepping methods with global non-dimensional time. Because the flows are almost steady, RANS with turbulence models can provide enough reasonable results. Two $k$ - $\omega$-models, SST and WD+, are applied to the prediction of the turbulent flows.

\section{Turbulence Models}

\subsection{Original $k-\omega$ model}

The original $k-\omega$ model developed by Wilcox ${ }^{(22)}$ is given as

$$
\begin{aligned}
& \frac{\partial \rho k}{\partial t}+\frac{\partial}{\partial x_{j}}\left(\rho u_{j} k-\left(\mu+\sigma_{k} \mu_{\mathrm{t}}\right) \frac{\partial k}{\partial x_{j}}\right)=\tau_{i j} \frac{\partial u_{i}}{\partial x_{j}}-\beta^{*} \rho k \omega, \\
& \frac{\partial \rho \omega}{\partial t}+\frac{\partial}{\partial x_{j}}\left(\rho u_{j} \omega-\left(\mu+\sigma_{\omega} \mu_{\mathrm{t}}\right) \frac{\partial \omega}{\partial x_{j}}\right)=\frac{\gamma \omega}{k} \tau_{i j} \frac{\partial u_{i}}{\partial x_{j}}-\beta \rho \omega^{2},
\end{aligned}
$$


where $\tau_{i j}=\mu_{\mathrm{t}}\left(2 S_{i j}-\frac{2}{3} \frac{\partial u_{k}}{\partial x_{k}} \delta_{i j}\right)-\frac{2}{3} \rho k \delta_{i j}, S_{i j}$ is the strain rate defined as $\left(\partial u_{i} / \partial x_{j}+\partial u_{j} / \partial x_{i}\right) / 2$.

The constants are $\sigma_{k}=\sigma_{\omega}=0.5, \beta^{*}=0.09, \gamma=5 / 9$ and $\beta=3 / 40$. The linear eddy viscosity is defined as

$$
\mu_{\mathrm{t}, \mathrm{Wilcox}}=\rho k / \omega
$$

Eq. (3) was originally designed to accurately predict the boundary layer flows with zero pressure gradient.

\subsection{WD+ model}

The linear eddy-viscosity definition in Eq. (3) is known to be inadequate in the prediction of flow under adverse pressure gradients. The weakly nonlinear correction is proposed to produce asymptotic behavior of $C_{\mu}$ when $S_{i j}$ approaches infinity. In the WD+ model proposed by Durbin ${ }^{(20)}$, the equations remain the same as those in the original Wilcox's version. A new formulation of eddy viscosity $\mu_{t}$ with a weakly nonlinear correction is defined as

$$
\mu_{\mathrm{t}, \mathrm{WD}+}=\min \left[\rho k / \omega ; \rho a_{1} k / \sqrt{\left(\Omega^{2}+S^{2}\right) / 2}\right]
$$

where $\Omega^{2}=2 W_{i j} W_{j i}, S^{2}=2 S_{i j} S_{j i}$ and $W_{i j}=\left(\partial u_{i} / \partial x_{j}-\partial u_{j} / \partial x_{i}\right) / 2, a_{1}=0.31$. The correction behaves well in SWBLI simulations.

\subsection{SST model}

This model also adopts Wilcox's $k-\omega$ model, which is well behaved near solid walls and needs no low Reynolds number corrections. At the same time, it combines with the standard $k$ - $\varepsilon$ model (reformulated in a $k$ - $\omega$ style), which is relatively insensitive to free-stream values in the outer edge of the boundary layer and free stream. The switching is realized by a flow dependent blending function. The SST model ${ }^{(21)}$ also limits the eddy viscosity by forcing the turbulent shear stress to be bounded according to the turbulent kinetic energy inside boundary layers (a realizability constraint). This modification improves the model's performance on flows with strong adverse pressure gradients and separation.

Mathematically, the SST turbulence model equations adopt $k$-equation and the $\omega$ equation as

$$
\begin{gathered}
\frac{\partial \rho k}{\partial t}+\frac{\partial}{\partial x_{j}}\left(\rho u_{j} k-\left(\mu+\sigma_{k} \mu_{\mathrm{t}}\right) \frac{\partial k}{\partial x_{j}}\right)=\tau_{i j} S_{i j}-\beta^{*} \rho k \omega, \\
\frac{\partial \rho \omega}{\partial t}+\frac{\partial}{\partial x_{j}}\left(\rho u_{j} \omega-\left(\mu+\sigma_{\omega} \mu_{\mathrm{t}}\right) \frac{\partial \omega}{\partial x_{j}}\right)= \\
\frac{\gamma \omega}{k} \tau_{i j} \frac{\partial u_{i}}{\partial x_{j}}-\beta \rho \omega^{2}+2\left(1-F_{1}\right) \frac{\rho \sigma_{\omega 2}}{\omega} \frac{\partial k}{\partial x_{j}} \frac{\partial \omega}{\partial x_{j}}
\end{gathered}
$$

where the blending function $F_{1}$ is defined as

$$
F_{1}=\tanh \left\{\left[\min \left(\max \left(\frac{\sqrt{k}}{0.09 \omega d} ; \frac{500 \mu}{\rho d^{2} \omega}\right) ; \frac{4 \rho \sigma_{\omega 2} k}{C D_{k \omega} d^{2}}\right)\right]^{4}\right\}
$$


and the cross diffusion term $C D_{k \omega}$ is defined as

$$
C D_{k \omega}=\max \left(\frac{2 \rho \sigma_{\omega 2}}{\omega} \frac{\partial k}{\partial x_{j}} \frac{\partial \omega}{\partial x_{j}} ; 10^{-20}\right) .
$$

Here, $\beta^{*}=0.09, \kappa=0.41 . d$ refers to the distance from the nearest wall. Some other constants are calculated from $\phi=F_{1} \phi_{1}+\left(1-F_{1}\right) \phi_{2}$, where the $\phi$ are the constants: $\sigma_{k 1}=0.85$, $\sigma_{\omega 1}=0.5, \beta_{1}=0.075, \gamma_{1}=\beta_{1} / \beta^{*}-\sigma_{\omega 1} \kappa^{2} / \sqrt{\beta^{*}}=0.553 ; \sigma_{k 2}=1.0, \sigma_{\omega 2}=0.856, \beta_{2}=0.0828$ $\gamma_{2}=\beta_{2} / \beta^{*}-\sigma_{\omega 2} \kappa^{2} / \sqrt{\beta^{*}}=0.44$

The eddy-viscosity coefficient in SST model is defined as

$$
\mu_{\mathrm{t}, \mathrm{SST}}=\min \left[\rho k / \omega ; \rho a_{1} k / \Omega F_{2}\right]
$$

where $F_{2}$ is another blending function defined as

$$
F_{2}=\tanh \left\{\left[\max \left(2 \frac{\sqrt{k}}{0.09 \omega d} ; \frac{500 \mu}{\rho d^{2} \omega}\right)\right]^{2}\right\}
$$

In Eq. (7), the eddy viscosity in SST model is similar in a form to that of Eq. (4) but also includes nonlinear effect. Therefore, the SST model can be thought as a weakly nonlinear turbulence model.

\section{Results and Discussions}

In order to deeply investigate the control effect of VGs, the flows past a single VG on a flat plane and a standard model an ONERA-M6 wing without any VGs are predicted to compare with available experimental data. At the end of this article, the effects on the flow-fields with and without a row of VGs on a supercritical airfoil and a wing are explored.

\subsection{Single VG on plane}

Yao, et al. ${ }^{(5)}$ presented detailed downstream flow-field measurements of a single vane-type low-profile VG in the Langley 20 by 28 Inch Shear Flow Tunnel. Here, the measurements around VG are applied to validate our code and investigate the flow features behind it.

As in Fig. 2, The deflection angle $(\alpha)$ of the $\mathrm{VG}$ is $16^{\circ}$ and the streamwise velocity is 34 $\mathrm{m} / \mathrm{s}$. The VG's length $(L)$ is $49 \mathrm{~mm}$, and its height $(H)$ is $7 \mathrm{~mm}$. Reynolds number based on $H$ is about 20000. The thickness $(d)$ of the VG could be ignored because it is just ten percent of $H$. The baseline flow is a typical freely developed boundary-layer flow, with VG installed at the place where the boundary layer thickness $(\delta)$ is $35 \mathrm{~mm}$. As VG's height is only about $20 \% \delta$ here, it is completely submerged in the boundary layer. This indicates that RANS is the suitable turbulence modeling method as no massive separation exists. If LES method is applied, a plenty of cells are needed in boundary layer at this high Reynolds number.

About 1.5 million cells, as shown in Fig. 3, are generated around VG with four-block point-to-point structured grids. The grids in the wake are clustered to predict more flow features. The boundary conditions are also labelled as inflow, outflow, symmetry and wall. The vortices past VG are also presented in Fig. 3. Due to the large deflection angle, the flow cannot keep attached on the leeward of the VG. Strong longitudinal vortices are detached 
from the leading edge of VG and they affect the main flow along a very long distance (such as $x / H=109)$ in the streamwise direction.

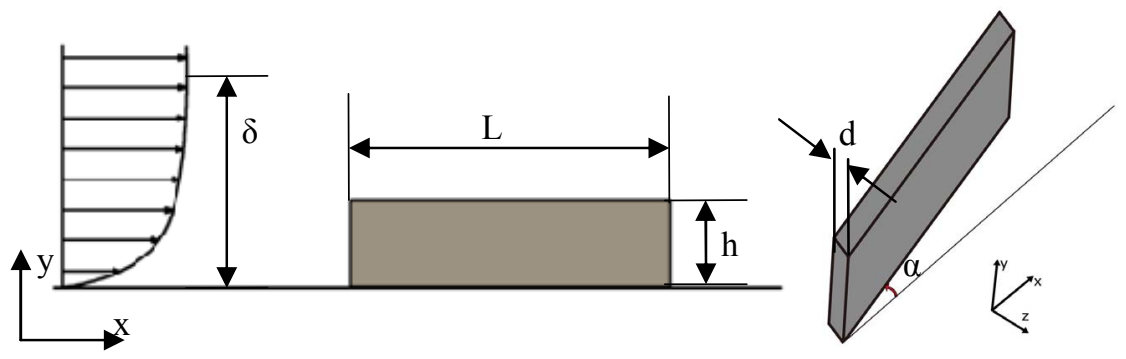

Figure 2 VG parameters.

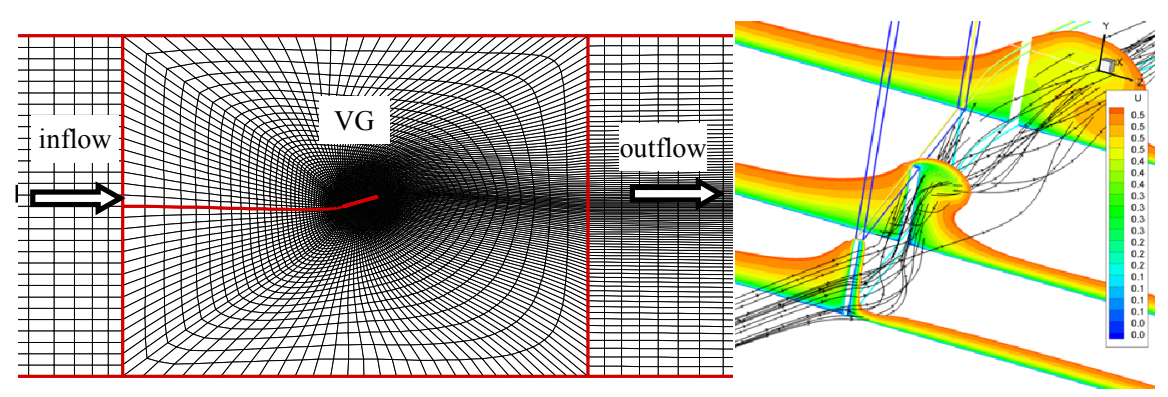

Figure 3 Grids and flow near the single VG.

RANS with SST model is applied to investigate the flow. The convection terms are dispersed using Roe scheme with the 3rd-order MUSCL and the 5th-order WENO interpolations, respectively. The results clearly present the differences between these two interpolations on the distribution of velocity and vorticity.

Comparisons of the streamwise velocities and vorticities between measurements and computations at six streamwise sections, where $x / H$ are 3, 10 and 109 after the trailing edge of VG, respectively, are plotted in Figs. 4 and 5. The vortices generated by VG are observed to give a continuous influence on the primary flow. At section $x / H=3$, the vortex is strong and concentrated with high value of streamwise vorticity $\left(\Omega_{x}\right)$ and the vortex core is near flat plane surface; as $x / H$ increases, the intensity of vortex weakens and the spanwise position of vortex core swifts outside; at section $x / H=109$, the vortex diffused with an unclearly seen core position. The difference between MUSCL and WENO shows that higher-order numerical scheme produces more accurate flow fields. The vortex at section $x / H=109$ almost disappeared by the 3rd-order MUSCL scheme, while the vortex height matches experiment data confidently by the 5th-order WENO scheme.

\subsection{SWBLI on ONERA-M6 wing}

A widely applied standard test case for 3D SWBLI prediction validation-ONERA-M6 wing case is calculated in transonic conditions, with Mach number $M_{\infty}=0.8447$, angle of attack $\alpha=5.06^{\circ}$ and Reynolds number based on mean aerodynamic chord $R e=11.7 \times 10^{6}$. The main difficulties of this case are the presentation of the " $\lambda$ " shock wave, the interactions of the shockwave with boundary layer and the separation induced by the strong shock. C-H type grids with 187 (streamwise) $\times 56$ (spanwise) $\times 65$ (normal) nodes and about $1 \times 10^{-5}$ first wall grid distance are used. 

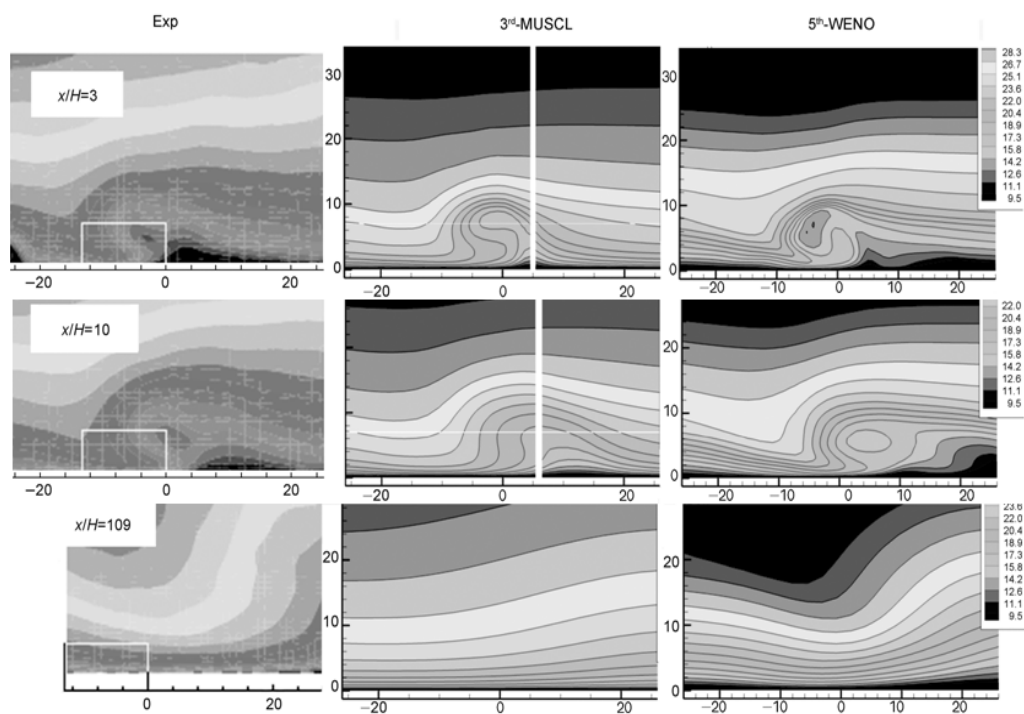

Figure 4 Comparisons of streamwise velocities $(\mathrm{m} / \mathrm{s})$ at 3 streamwise stations.

Exp
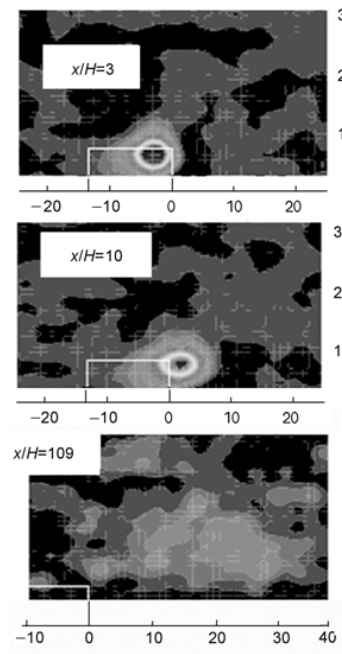

$3^{\top+-M U S C L}$
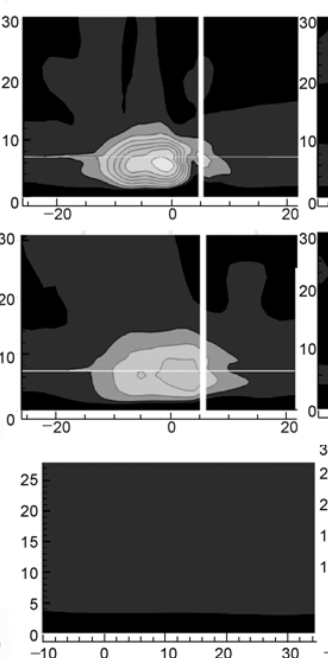

$5^{\text {m}}$-WENO
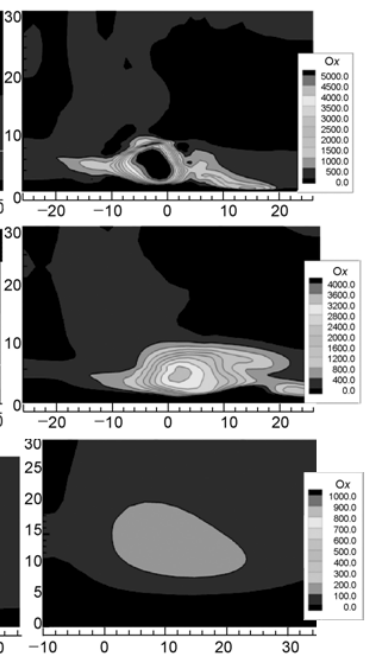

Figure 5 Comparisons of streamwise vorticity components (1/s) at 3 streamwise stations.

The pressure coefficients are compared on the wing surface at $2 z / B=22 \%, 44 \%, 65 \%$, $80 \%, 90 \%$, and $95 \%$, shown in Fig. 6. Results by both turbulence models match the measurements very well and the differences are quite small. It is observed that the predicted shock position with SST model is a little more upstream than that with WD+ model, especially near the wing tip. The difference may reflect the different definitions of the eddy viscosity and the closure coefficients in the two models.

\subsection{VGs on a supercritical aifoil in transonic case}

In transonic case, strong shockwave/boundary-layer interactions occur at some angle of attack. To investigate the effect of VGs on a supercritical wing, we will firstly predict the simplified case in this section, with the wing replaced by an infinite-width supercritical airfoil. The configuration is shown in Fig. 7. Row of VGs with a deflection angle of 12 degrees to the leading edge mounted at $21 \%$ chord streamwise position, are distributed 
evenly with a spanwise space(w) of $6 \mathrm{VG}$ height(h). The VG plates are rectangular in shape with height/length ratio of 1:5. The height $\mathrm{h}$ is 0.003 chord length(c).
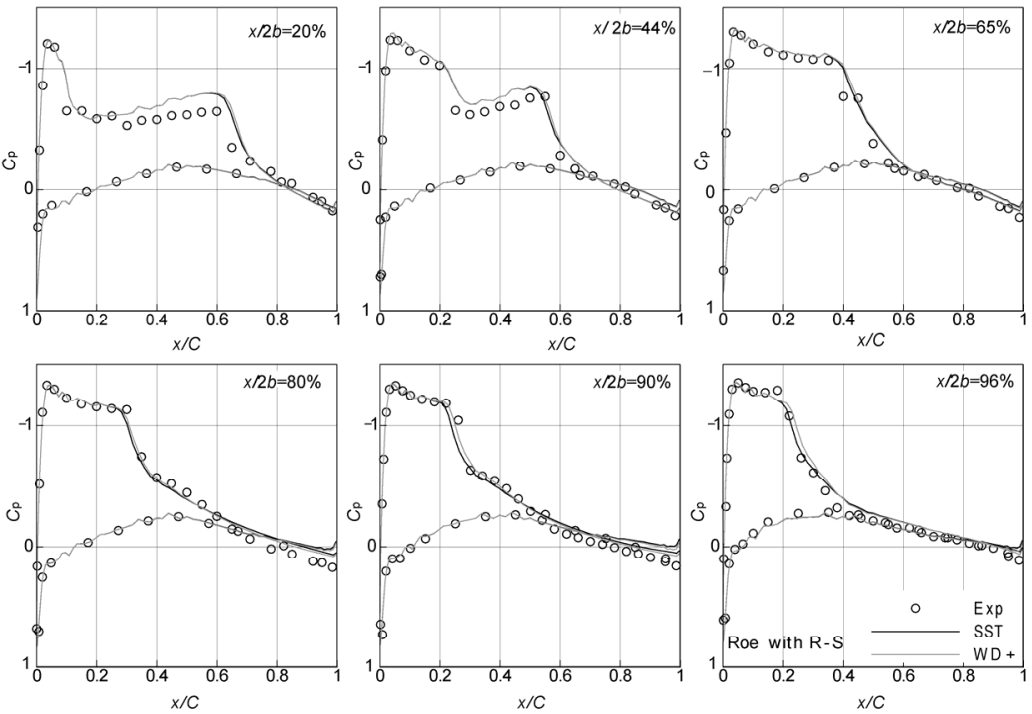

Figure 6 Comparisons of spanwise pressure coefficients.

In favor of the periodicity of the configuration, the computational region includes only one VG with a spanwise width $w=0.018 \mathrm{c}$. 451 grids on the wing surface and 41 points in the spanwise direction are generated. Grids near VG are refined to catch vortex structures behind it. Free-stream Mach number is chosen as 0.725 , the angle of attack is 4 degrees, and Reynolds number based on $\mathrm{c}$ is $2.2 \times 10^{7}$.

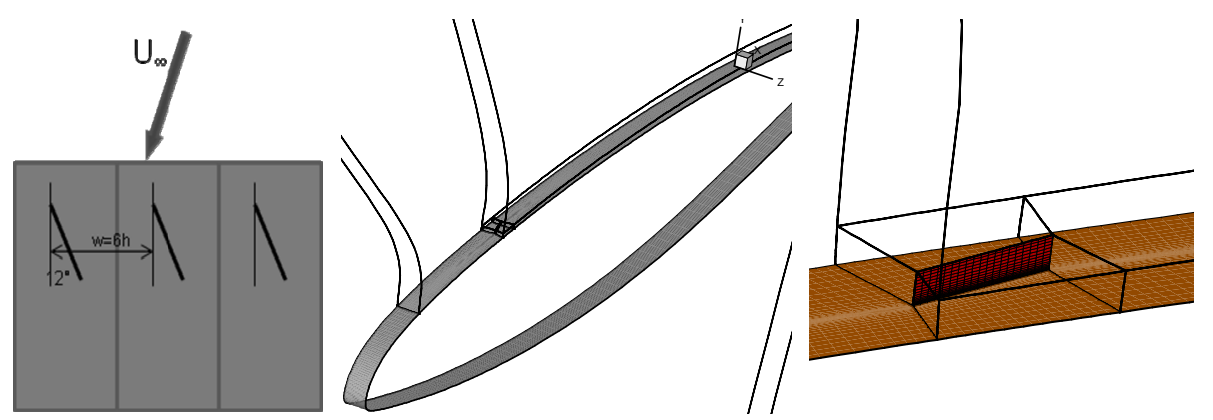

Figure 7 Configuration and grids near VG plate on the airfoil.

Spanwise slice of time-averaged flow fields with and without VGs are shown in Fig. 8. In uncontrolled case, separation bubble exists behind shockwave region, and stretched through until near trailing edge. In controlled case, however, the separation bubble becomes half the height, and are suppressed within small regions. In Fig. 9, flow fields are repeated 4 times in spanwise direction for a clear display. The streamwise vortex can be clearly seen to intercat with shockwave structure. From the Cp slice, oscillation exists due to the separation bubble in uncontrolled case.

In fact, the uncontrolled case is unsteady with shockwave position shifting front and back. The controlled one is steady, though with value of lift and drag unchanged too much. The VGs have suppressed the separation phenomena and the whole flow fileds in this transonic case.

\subsection{VGs on a supercritical wing with strong SWBLIs}

3-dimension effect of VGs on a supercritical wing are investigated in this section, with 
the same cross-section airfoil design predicted in the previous section.
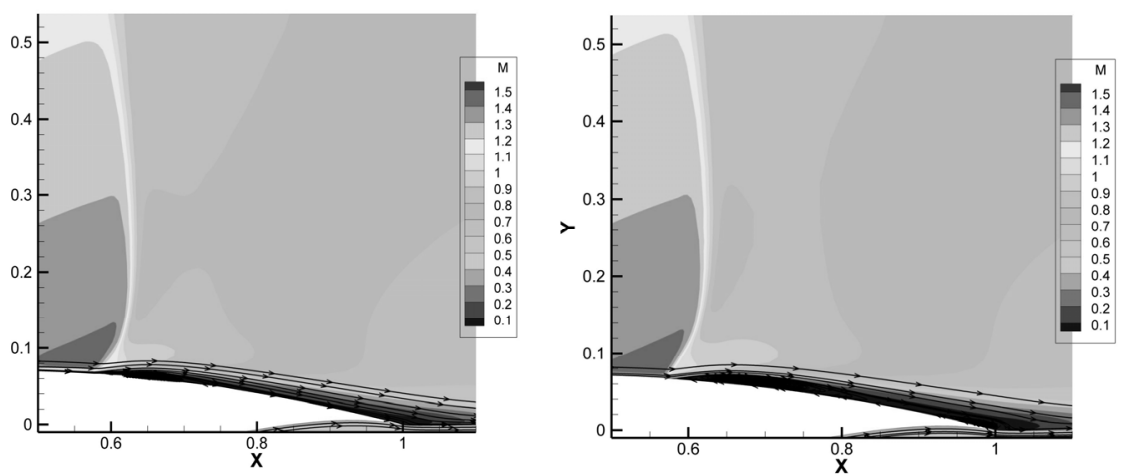

Figure 8 Field slice with and without VGs.
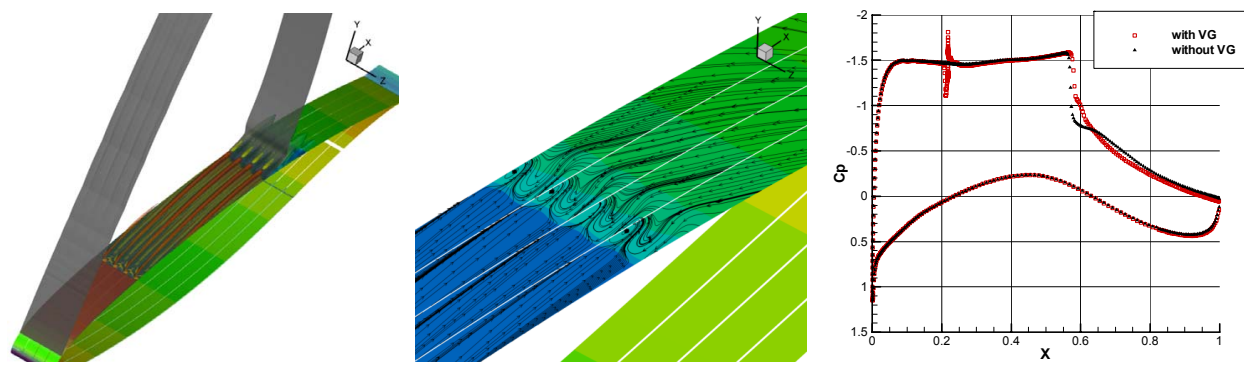

Figure 9 Flow field with 4 VGs(Left: Shockwave filed; Middle: Surface streamlines near shockwave; Right: $\mathrm{Cp}$ slice).

Computational efficiency contradiction occurs in numerical prediction of VG flows, as the size of VG is almost the same as boundary layer thickness and it is much smaller than the mean aerodynamic chord (MAC, about 1\%). In order to predict the wake flow, clustering and high-quality grids are needed behind VGs. If the point-to-point (PtoP) grids are applied, several millions of cells are required. Appropriate grids are required to accurately simulate viscous area in boundary layer and the narrow long wake region after VGs.

The baseline wing configuration and the grids are presented in Fig. 10. Two blocks of PtoP grids (with $151 \times 101 \times 81+66 \times 11 \times 26 \cong 1.25$ million cells) are applied to describe the flow fields around the original configuration. Cell height of the first grid from the wall in viscous layer is $2 \times 10^{-5} \mathrm{MAC}$.

A row of VGs (maximum number is seven) is laid at about 25\% MAC in streamwise direction, shown in Fig. 10. All the VG slices are rectangle with size of $66 \mathrm{~mm} \times 17 \mathrm{~mm} \times 2$ $\mathrm{mm}$, aligned from $2 z / B=43.9 \%-70.6 \%$ in the spanwise direction and at $24.25 \%$ chord in the streamwise direction. They are evenly spaced and the deflection angle is $25^{\circ}$. Because the thickness over height is about 0.1 , the thickness is ignored.

In order to satisfy the computational accuracy and efficiency, 3.22 million Quasi-PtoP grids with inner blocks densified by 2 or 3 times in one direction, are generated (see Fig. 10). Near VGs, the grids, named inner grids, are locally clustered and PtoP. Far away from VGs, PtoP but much coarser grids, named outer grids, are applied. At the interface between the inner and outer grids, Quasi-PtoP grids are applied. Grid density ratios of inner VG blocks to outer blocks are 3:1 in the spanwise direction and 2:1 in both streamwise and normal directions. As the VG thickness could be ignored, it is very convenient to set the VGs' number. If VG is turned on, the boundary condition at the VG is set as wall. If VG is turned off, the boundary condition is set as inner cells of the continuous flow fields without 
any wall. In this work, cases of 2, 3 and 7 VGs are investigated.

The flow is typical transonic flow where the cruise Mach number is 0.785 and the Reynolds number is $5.94 \times 10^{6} / \mathrm{m}$. To investigate the VG effects on SWBLI, the angle of attack is taken as $4^{\circ}$. Due to a little large angle of attack, the flow is dominated by separation induced by strong SWBLI. The turbulence model is taken as two-equation $k-\omega-\mathrm{WD}+$ model.

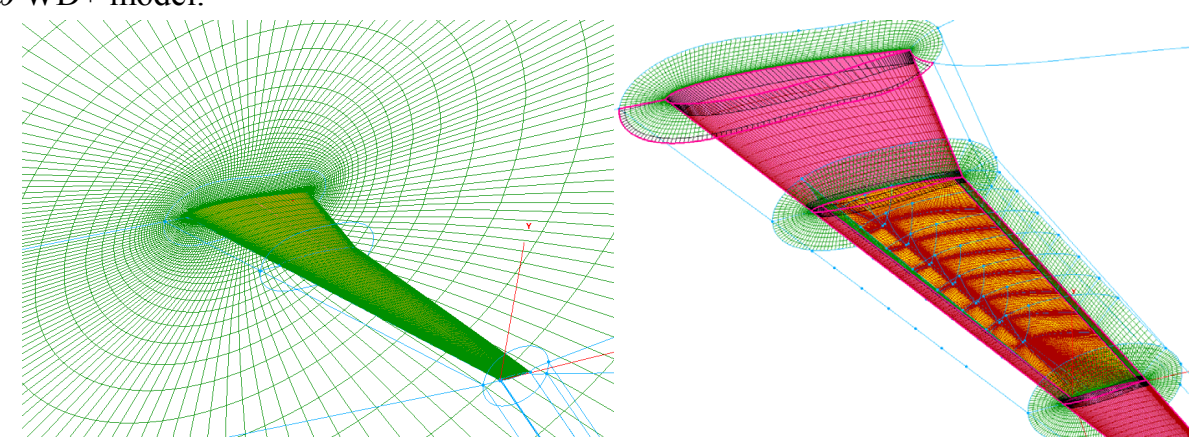

Figure 10 Grids around the supercritical wing and VGs.

The pressure contour and streamlines on the upper surface of the wing without and with 2, 3 and 7 VGs are presented in Fig. 11.

- Without VG: Strong shockwave is observed on the upper wing, and boundary layer separates after it forms a high-pressure region, which decreases lift. Spanwise flow looks very intense after separation, and accumulates upon wing tip. A stronger wing tip vortex forms and more energy consumes, which will result in drag increase. For these reasons, strong boundary layer separation should be avoided in wing design. In addition, flow after shockwave separation is always unsteady and may cause buffet, which is a harmful phenominon in flight.

- 2 VGs: Spanwise flow is blocked in the VG region. The inner VG holds up the spanwise vortice development and a bump of vortices are accumulated after it. However, outside the VG region through wing tip, spanwise flow is still intense.

- 3 VGs: Similar to 2 VGs' case, spanwise flow is weakened in the VG region with relatively small wing tip separation.

- 7 VGs: In all the test cases, 7 VGs result in the best control effect of upper wing surface SWBLIs. Spanwise flow is effectively weakened, with no clear accumlation of vortice bump. Outside the VG regions around wing tip, there are weakend spanwise separations.

The spatial vortices and streamlines are presented in Fig. 12, expressed by isosurface of 2-order invariants of velocity tensor $Q=0.5 \times\left(S_{i j}-W_{i j}\right)=-10$.

- No VG: Outside of the kink, spanwise vortices over the clear wing are formed through wide ranges. Strong wing tip vortex exists.

- 2 VGs: Spanwise vortices and wing tip vortex are weakened. However, 2 vortex bumps are formed around $2 \mathrm{VG}$ surfaces.

- 3 VGs: Separations and wing tip vortex are further reduced. However, vortex bump still exists around the inner VG.

- 7 VGs: Upper wing suraface separation and spanwise flow are almost reduced. Only weak separation exists after the VG regions. 

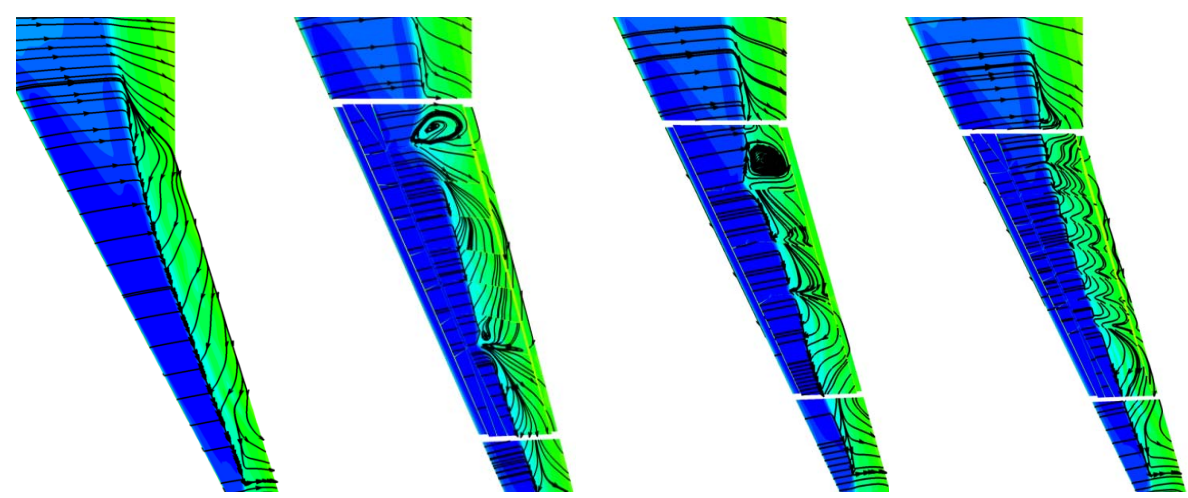

Figure 11 Surface streamlines over the wing without VG and with 2, 3 and 7 VGs.

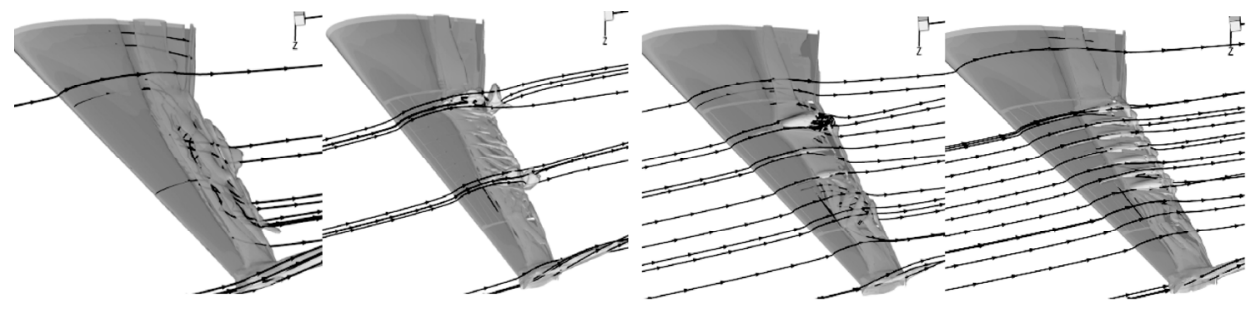

Figure 12 Vortices and streamlines over the wing with no VG and 2, 3, 7 VGs.

Then, we can conclude that VGs can effectively reduce the separations behind the shock wave. For maximum VGs numbers ( 7 here), the controled flows are desired.

To investigate force effects of VGs, averaged coefficents of lift and drag and ratio of $C_{1}$ to $C_{\mathrm{d}}$ are listed in Table 1 .

Table 1 Coefficents of lift and drag and ratio of $C_{1}$ to $C_{\mathrm{d}}$

\begin{tabular}{lcccc}
\hline & No VG & 2 VGs & 3 VGs & 7 VGs \\
\hline$C_{\mathrm{l}}$ & 0.6728 & 0.6571 & 0.6516 & 0.6592 \\
$C_{\mathrm{d}}$ & 0.0416 & 0.0411 & 0.0404 & 0.0406 \\
$C_{\mathrm{l}} / C_{\mathrm{d}}$ & 16.17 & 16.00 & 16.12 & 16.24 \\
\hline
\end{tabular}

- 2 VGs: Drag decreases although VG slices add additional aerodynamic surfaces into the main flow. It can be concluded that separation reduction in mid-wing region decreases drag. However, lift is also decreased.

- 3 VGs: Both lift and drag decrease.

- 7 VGs: Drag is similar to 3 VGs' case. But lift is higher than 3 VGs' case, evidently resulting from the stablized flow separation. The ratio of $C_{1}$ to $C_{\mathrm{d}}$ is even higher than baseline wing case. The overall control effect is the best in all the cases.

\subsection{Low-speed high angle of attack VG flow of a supercritical wing}

The flow with VGs on the upper wing surface in the low-speed conditions is also investigated. At high angles of attack with Mach number of 0.2, massive separation occurs near the leading edge. In this article, angle of attack is taken as $16^{\circ}$.

Surface streamlines and isosurface of $Q$ are given in Fig. 13. Separation region stretches until about halfway the spanwise location in baseline case. The VG-configurations are the same as those in transonic cases.

In fact, if VGs are assigned at $24.25 \%$ chord length, they do not show any improvement on separations. The reason is that VGs are submerged completely in massive separation regions, compared to flow separation in transonic cases shown before. Within separated 
boundary layers, small-scale VGs could not function correctly and induce no useful wake vortex.

Then, VGs are moved forward to $3.5 \%$ chord-aerodynamic chord which is very close to the leading edge. VGs now function well, and the separation region is reduced now to near wing tip (see Fig. 12).

\section{Conclusion}

This article investigates VG's control effect on a supercritical wing with Quasi-PtoP structured grids. The flows past a single VG on plane and ONERA-M6 are predicted to obtain basic knowledge of VG and SWBLI flow features. The comparisons between baseline wing and 2, 3 and 7 VGs have shown that VGs on the wings in the transonic condition can effectively suppress the spanwise flow and separations behind the shock wave. 7 VGs show best control effects of boundary-layer separation and induced spanwise flows. For low-speed case, small-scale VGs will function better at locations before flow separations. Otherwise it will cause even worse drag increase. After all, more investigations into the VG model and the application are still probably needed.

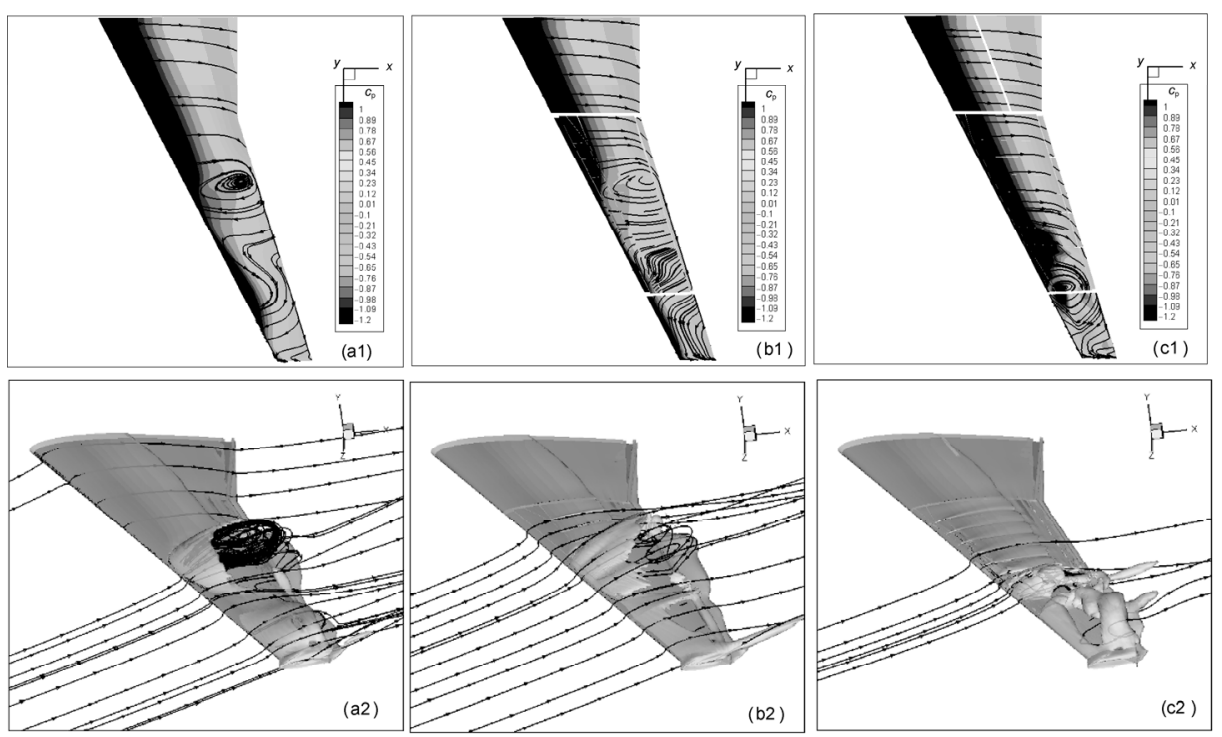

Figure 13 Surface streamlines and isosurfaces of $Q$ at $16^{\circ}$ AOA in the low-speed condition. (a1) (a2) No VG; (b1) (b2) 7 VGs at $24.25 \%$ streamwise location; (c1) (c2) 7 VGs at leading edge.

\section{Acknoledgement}

This work was supported by the National Natural Science Foundation of China (Grant No. 10932005).

\section{References}

(1) Vos J. B., Rizzi A., Darracq D. and Hirschel E.H., Navier-Stokes solvers in European aircraft design, Progress in Aerospace Sciences, Vol. 38, No. 8 2002, pp. 601-697.

(2) Liu G., Liu W., Mou B. and Xiao Z.Y., CFD numerical simulation investigation of vortex generators, Acta Aerodynamica Sinica, Vol. 25, No. 2 2007, pp. 241-244.

(3) Ni Y. Q., Develoment of the vortex-generator and study on the effect of vortex-generator on boundary layer, Acta Aerodynamica Sinica, Vol. 13 1995, pp. 110-116.

(4) Lin J. C., Review of research on low-profile vortex generators to control boundary-layer separation, Progress in Aerospace Sciences, Vol. 38 2002, pp. 389-420.

(5) Yao C. S., Lin J. C. and Allan B. G., Flow-field measurement of device-induced embedded streamwise vortex on a flat plate, AIAA, 2002-3162.

(6) Allan B. G., Yao C. S. and Lin J. C., Simulation of embedded streamwise vortices on a 
flat plate, NASA/CR-2002-211654, ICASE Report, 2002-14.

(7) Jirasek A., A vortex generator model and its application to flow control, AIAA, 2004-4965.

(8) Erik W. and Scott T. S., Numerical simulation of micro vortex generators, AIAA, 2004-2697.

(9) Zhang J. and Zhang B. Q., Investigation of boundary layer separation control for supercritical airfoil using micro vortex generator (in Chinese), Journal of Experiments in Fluid Mechanics, Vol. 19, No. 3 2005, pp. 94-97.

(10) Valilis P. S. and Ekaterinaris J. A., Computational investigation of flow control over wings, AIAA, 2007-477.

(11) Stillfried F., Lögdberg O., Wallin S. and Johansson A., Statistical modeling of the influence of turbulent flow separation control devices, AIAA, 2009-1501.

(12) Xiao Z. X., Study of complex flow by solving NS equations and application of turbulence models (in Chinese), Doctoral Dissertation (2003), Northwestern Polytech University.

(13) Xiao Z. X., RANS/LES hybrid method based on aircraft design (in Chinese), Postdoc Final Report (2005), Tsinghua University.

(14) Xiao Z. X., Chen H. X., Zhang Y. F., Huang J. B. and Fu S., Study of delayed-detached eddy simulation with weakly nonlinear turbulence model, Journal of Aircraft, Vol. 43 2006, pp. 1377-1385.

(15) Fu S., Xiao Z. X., Chen H. X., Zhang Y. F. and Huang J.B., Simulation of wing-body junction flows with hybrid RANS/LES methods, International Journal of Heat and Fluid Flow, Vol. 28 2007, pp. 13791390.

(16) Xiao Z. X., Zhang Y. F., Huang J. B., Chen H. X. and Fu S., Prediction of separation flows around a 6:1 prolate spheroid using RANS/LES hybrid approaches, Acta Mechanica Sinica, Vol 23, No. 4 2007, pp. 369-382.

(17) Xiao Z. X. and Fu S., Studies of the unsteady supersonic base flows around three afterbodies, Acta Mechanica Sinica, Vol. 25, No. 4 2009, pp. 471-479.

(18) Radesipel R. and Swanson R. C., Progress with multi-grid schemes for hypersonic flow problems, NASA Contractor Report No. 189579, 1991.

(19) Soo H. P. and Jang H. K., Implementation of $k-\omega$ turbulence models in an implicit multigrid method, AIAA Journal, Vol. 42, No. 7 2004, pp. 1348-1357.

(20) Durbin P. A., On the $k-\varepsilon$ stagnation point anomaly, International Journal of Heat and Fluid Flow, Vol. 17, No.1 1996, pp. 89-90.

(21) Menter F. R., Two-equation eddy-viscosity turbulence models for engineering applications, AIAA Journal, Vol. 32 1994, pp. 1598-1605.

(22) Wilcox D. C., Reassessment of the scale-determining equation for advanced turbulence models, AIAA Journal, Vol. 26, No. 11 1988, pp. 1299-1310. 